Presented at IEEE Nucl. Sci. Symp., Orlando, FL

Oct. 27-31, 1992

CQR'ES

BNL --48456

DE93 009346

\title{
A Study on the Use of Lead Fluoride for Electromagnetic Calorimetry
}

\author{
C.L.Woody, J.A.Kierstead, P.W.Levy, S.Stoll, A.B.Weingarteri \\ Brookhaven National Laboratory \\ D.F.Anderson and F.J.Ramberg
}

Fermilab

Y.Kuno, J.A.Macdonald, A.Konàka, D.A.Hutcheơn

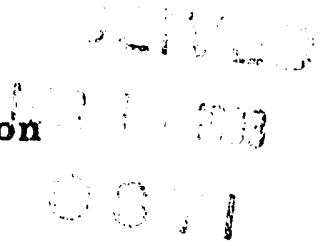

\section{$A B S T R A C T$}

A study has been made on the properties of lead fluoride as a Cherenkov material for use in electromagnetic calorimetry. A prototype calorimeter module consisting of a $5 \times 5$ array of $2.1 \times 2.1 \times 18.5 \mathrm{~cm}^{3}$ crystals has been built and tested in a test beam at the Brookhaven AGS. Results are given on energy resolution, shower sixe and $e / \pi$ separation for electrons and pions in the range from 1-4 GeV. The light output has been measured to give $Z 1000$ photoelectrons per MeV in good quality crystals, and to provide useful signals down to as low as $32 \mathrm{MeV}$. Measurements were also made on radiation damage in lead fluoride using ${ }^{60} \mathrm{Co}$ gamma rays and high energy ionizing particles, as well as on thermoluminescence after irradiation. It was found that only modest damage occurs up to a level of $\sim 30 \mathrm{Krad}$ in large, calorimeter size crystals, and that the damage can be easily removed by optical bleaching.

\section{INTRODUCTION}

Lead fluoride $\left(\mathrm{PbF}_{2}\right)$ is newly rediscovered Cherenkov material [1-2] with very high density $\left(7.77 \mathrm{~g} / \mathrm{cm}^{3}\right)$, a short radiation length $(9.3 \mathrm{~mm})$ and a small Moliere radius $(2.2 \mathrm{~cm})$. The Moliere radius is smaller than the densest commonly used scintillating crystal, BGO, and is effectively even smaller given that very soft particles in the periphery of a shower do not produce Cherenkov light. Due to its high density, it is an attractive candidate for use reactions where particle multiplicities are very high or where a high degree of compactness is desired. Being a Cherenkov material, it does not produce as much light as : cintillating crystal, but its light output is sufficiently high to give an energy resolution equal to or better than lead glass. In addition, it shows rather good radiation hardness, and has the promise of being able to be produced in large quantities at a reasonable cost.

\section{LEAD FLUORIDE CALORIMETER TESTS}

A prototype $P b F_{2}$ calorimeter consisting of a $5 \times 5$ array of $2.1 \times 2.1 \times 18.6 \mathrm{~cm}^{3}$ crystals (approximately one square Moliere radius by 20 radiation lengths) was built and tested in beams of electrons and pions in the momentum range from 1-4 GeV/c at the Brookhaven AGS. The crystals were produced by Optovac, Inc. [3] under an R\&D contract to develop large, calorimeter sized crystals with good UV transparency and high radiation hardness. Approximately half of the crystals in the detector were of excellent quality and had good optical transmission extending down below $300 \mathrm{~nm}$, as shown in Figure 1 . Others, which were grown in early attempts to produce large crystals, were of poorer quality and were placed around the outside edges of the calorimeter. New crystals are being produced by Optovac to replace the older ones, but they unfortunately did not arrive in time for our besm tests.

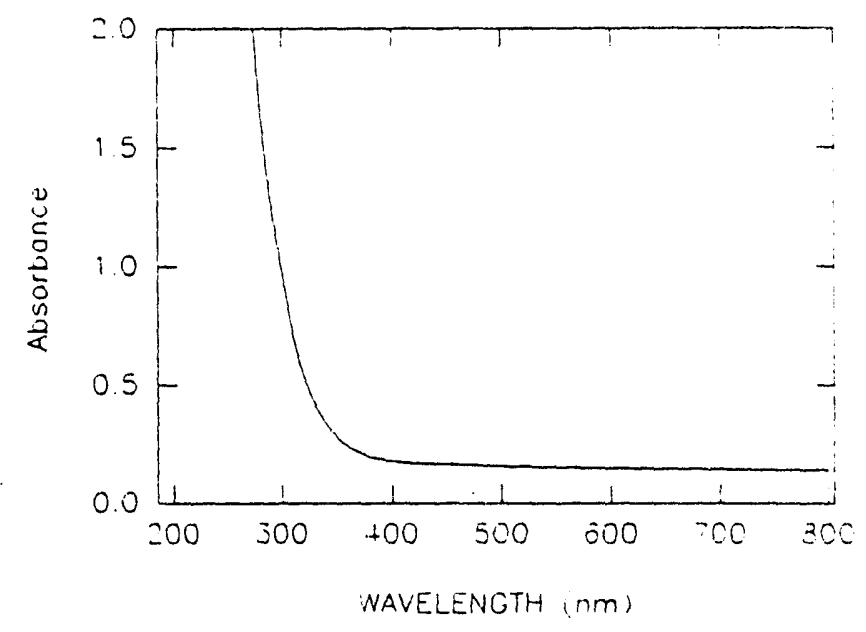

Fig. 1: Absorbance vs wavelength for a bigh quality crystal used in the $5 \times 5$ calorimeter array. 
Table I

Measured Energy Resolution Before Gain Balancing

\begin{tabular}{|c|c|c|}
\hline Energy (GeV) & $\begin{array}{c}\sigma / E \\
\text { central } 3 \times 3\end{array}$ & $\begin{array}{c}\sigma / E \\
\text { total } 5 \times 5\end{array}$ \\
\hline 1 & $5.9 \%$ & $5.6 \%$ \\
\hline 2 & $4.1 \%$ & $3.9 \%$ \\
\hline 3 & $3.4 \%$ & $3.2 \%$ \\
\hline 4 & $3.4 \%$ & $3.5 \%$ \\
\hline
\end{tabular}

The crystals were wrapped in white reflecting teflon and read out with $3 / 4^{n}$ UV glass window photomultiplier tubes (Hamamatsu R3479) which were coupled to the crystals with a UV transmitting optical grease (GE Viscasil $600 \mathrm{M}$ ). The photocathode covered approximately $40 \%$ of area of the readout end of the crystals. The number of photoelectrons per $\mathrm{GeV}$ was measured by determining the peak position for minimum ionizing particles in each crystal $\left(E_{\text {dep }} \sim 179 \mathrm{MeV}\right)$ along with an independent gain calibration for each phototube using an LED. The best crystals gave yields between 1000-1100 photoelectrons per $\mathrm{GeV}$, while the average number of varied from $\sim 900$ for the central nine crystals to $\sim 720$ for the outer sixteen crystals.

The analysis of the data from the test beam run is not yet complete, but some preliminary results have been obtained. The energy resolution, as determined by fitting the raw data over the peak of the electron energy spectrum, but excluding the radiative tail caused by energy loss of the electrons due to material upstrean in the beam, is given in Table I. The results are given separately for the central $3 \times 3$ array of crystals and the entire $5 \times 5$ array. The data have not been corrected for gain equalization of each channel, nor for run to run gain variations. As a result, the $4 \mathrm{GeV}$ point has either no better or slightly worse resolution than the $3 \mathrm{GeV}$ point, and requires further corrections before it can be fit with the other data to determine the energy dependence of the resolution. A fit of the data from 1-3 GeV gives a resolution of $\sigma / E=5.95 \% / \sqrt{E}$ for the $3 \times 3$ array, and $\sigma / E=5.70 \% / \sqrt{E}$ for the $5 \times 5$ axray. In both cases, the constant term was consistent with zero $(\sim \pm 0.1 \%)$, and the noise term, which was determined by measuring the widths for the sum of pedestals for the $3 \times 3$ and $5 \times 5$ arrays, was found to be $0.2 \% / \mathrm{E}$ and $0.3 \% / \mathrm{E}$, respectively.

Data were taken with $4 \mathrm{GeV}$ pions passing down the center of each crystal in order to determine the gain equalization factors for each crystal and photomultiplier tube. In addition, a laser calibration system was used with the calorimeter to correct for drifts in these gain factors from run to run. Figure 2 shows the energy spectrum for $1 \mathrm{GeV}$ electrons, summed over the entire detector, after the gain equalization factors were applied. The gain

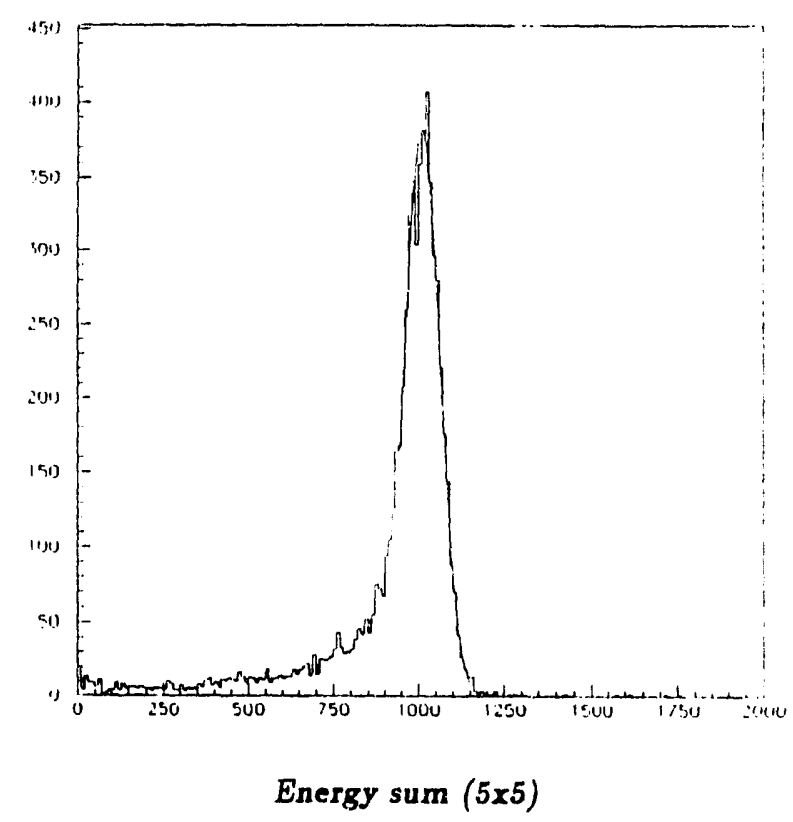

Fig. 2: Pulse height spectrum for $1 \mathrm{GeV}$ electrons after gain balancing of all photomultiplier tubes. The resolution obtained by fitting the spectrum over the pesk is $5.1 \%$.

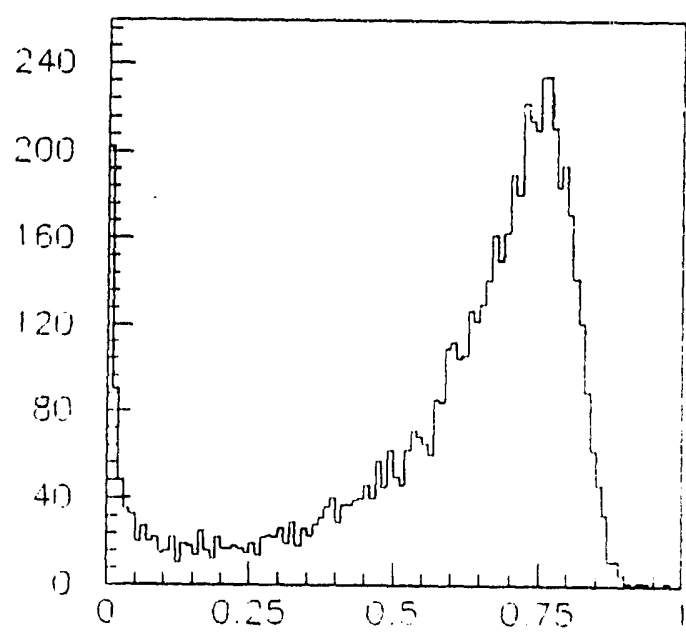

Energy fraction in central crystal

Fig. 3: Fraction of energy contained in the central crystal for an incident electron energy of 1 GeV.

equalisation improved the energy resolution from $5.6 \%$ to $5.1 \%$. The process of correcting the rest of the data with the gain factors and laser calibration is still under way, but is expected to result in an improvement in the energy resolution at other energies as well.

The electron data was also used to study the shower size in the detector. Figure 3 shows that the fraction of energy contained in the central crystal for an incident electron energy of $1 \mathrm{GeV}$ is approximately $75 \%$. It was found that more than $95 \%$ of the energy was contained in the central $3 \times 3$ array, and that these fractions did not vary significantly with energy from $1-4 \mathrm{GeV}$. This is 


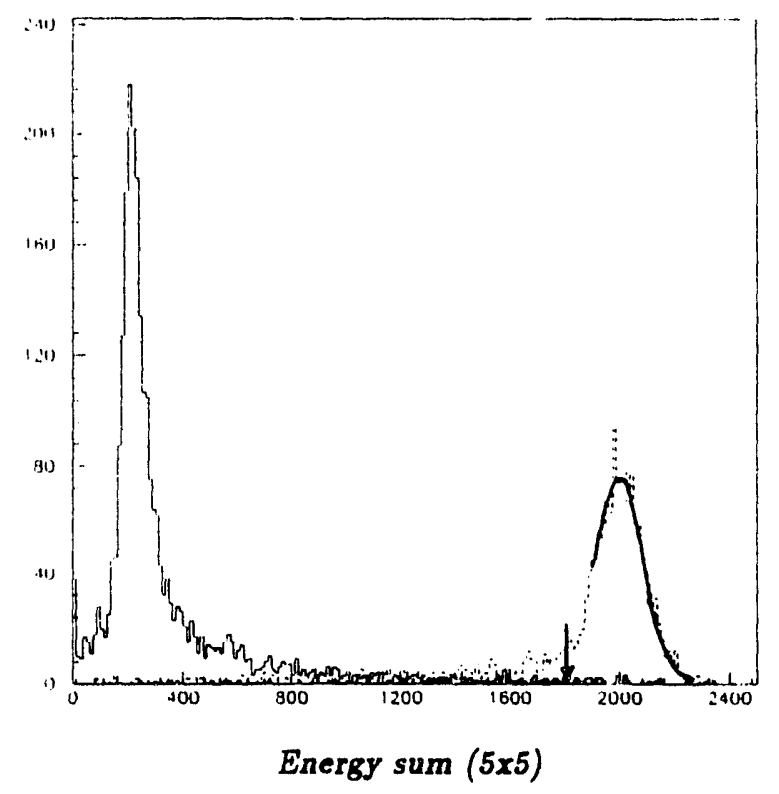

Fig. 4: Pulse height spectrum for $2 \mathrm{GeV}$ electrons and pions. The cut indicates an $e / \pi$ rejection of $1: 100$. Electron efficiency is discussed in the text.

consisteat with a Moliere radius in $P b F_{2}$ of $\$ 2 \mathrm{~cm}$. The position resolution was also measured using a logarithmic weighting procedure $[4]$, and determined to be on the order of a few millimeters.

The $e / \pi$ rejection factor was determined using electron and pion data at $2 \mathrm{GeV}$. Figure 4 gives the two spectra, showing a clear separation between the minimum ionising pion peak and the electron pesk. The electron spectrum again shows a large radiative tail due to energy loss caused by material upstream in the beamline. As a result, for an efficiency cut on the pion data at $\sim 1 \%(e / \pi \sim 1: 100)$, the apparent electron efficiency is only $\sim 80 \%$. However, if one fits the electron data over the peak, avoiding the tail, and takes a $2 \sigma$ cut, then the electron efficiency increases to $95 \%$.

The low energy response of $\mathrm{PbF}_{2}$ was measured with positrons in the momentum range from 32 to $147 \mathrm{MeV} / \mathrm{c}$ in a separate beam test at TRIUMF [5].For this test, only a single crystal was used which had an octagonal cross section ( $4.3 \mathrm{~cm}$ flat-to-flat by $13.3 \mathrm{~cm}$ long) and was of poorer quality than most of the crystals used in the calorimeter. It was wrapped with two layers of teflon tape and one layer of aluminired mylar, and coupled to a $2^{n}$ diameter quarts window photomultiplier tube (Phillips XP2020Q) with UV transmitting optical grease. The number of photoelectrons measured as a function position momentum is shown in Figure 5 . The slight decease in photoelectron yield at the higher energies is due to saturation in the phototube. The average number of photoelectrons per $\mathrm{GeV}$ was determined to be $\sim 1700$ for the full photocathode coverage provided by the $2^{n}$ phototube. This is somewhat less than the number which would be predicted for the better crystals used in

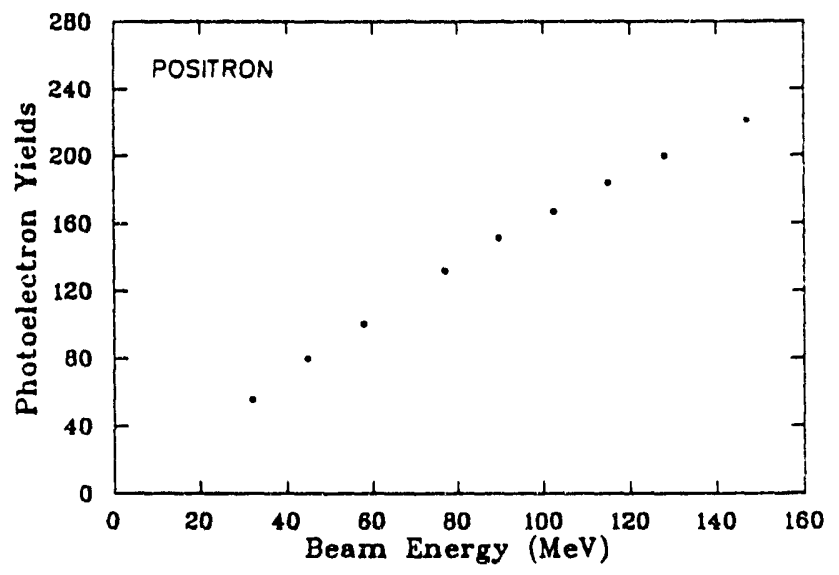

Fig. 5: Photoelectron yield as a function of positron momentum for a $13.3 \mathrm{~cm}$ long $P b F_{2}$ crystal.

the prototype calorimeter, but does demonstrate the low energy measuring capability of $P b . F_{2}$.

\section{RADIATION DAMAGE}

We have carried out a number of studies of radiation damage in $\mathrm{PbF}_{2}$, including irradiations with ${ }^{60} \mathrm{Co}$ gamma rays, high energy hadrons, and measuring thermoluminescence after irradiation. An example of the results on the change in ausorbance due to ${ }^{60} \mathrm{Co}$ radiation is shown in Figure 6. The absorbance, which was measured along the axis of the $18.6 \mathrm{~cm}$ long crystal within 20 minutes after each exposure, shows only modest damage up to a dose of $30 \mathrm{Krad}$, followed by more severe damage up to 1 Mrad.

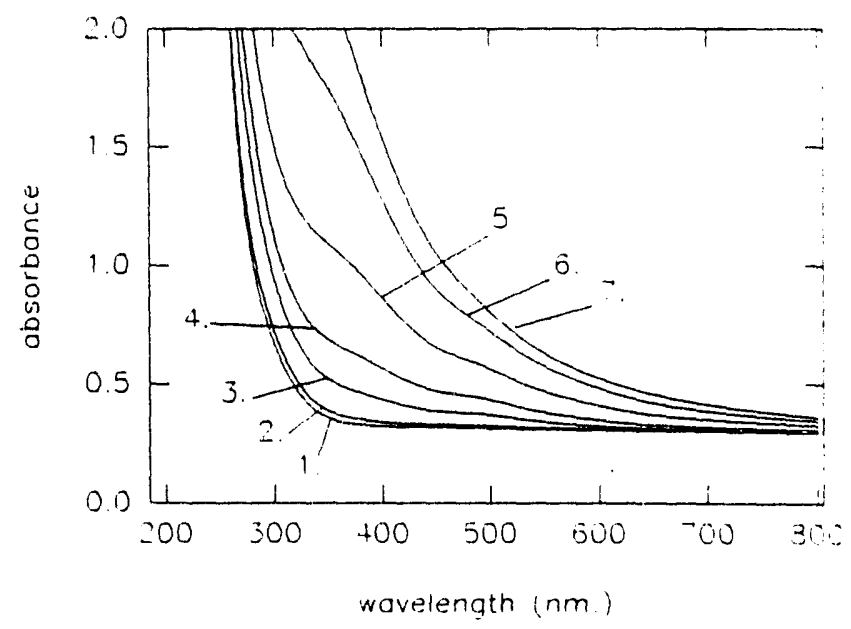

Fig. 6: Absorbance vs wavelength for various doses of ${ }^{60} \mathrm{Co}$ radiation for an $18.6 \mathrm{~cm}$ long $\mathrm{PbF}_{2}$ crystal. 1- unirradiated, 2- $10^{3} \mathrm{rad}, 3-10^{4} \mathrm{rad}, 4-$ $3 \times 10^{4} \mathrm{rad}, 5-10^{5} \mathrm{rad}, 6-3 \times 10^{5} \mathrm{rad}, 7-10^{6} \mathrm{rad}$. 


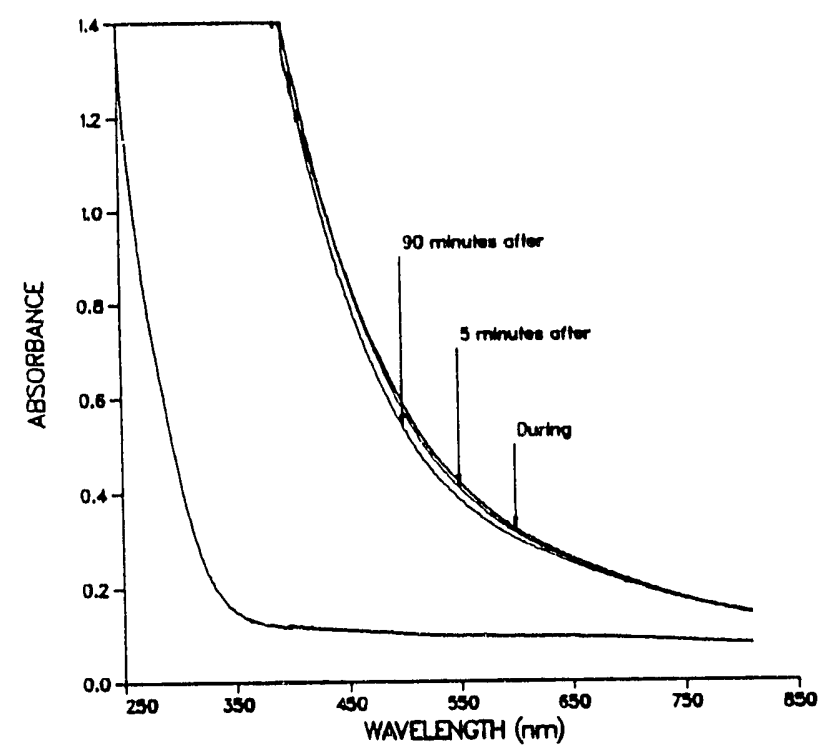

Fig. 7: Absorbance spectra for a 1" diameter $x 1$ "long $\mathrm{PbF}_{2}$ sample measured during and immediately after irradiation to $1.8 \mathrm{Mrad}$ with ${ }^{60} \mathrm{Co}$ gamma rays.

Although the amount of damage is still considerable, the new results represent a tremendous improvement over the initial samples which were first measured [1]. This improvement has mainly come about by improvements in the purity of the raw material and in the growth and processing techniques used to produce the crystals. It is likely that the remaining damage seen in $P b F_{2}$ is still due to residual impurities and/or defects, and that the radiation hardness will increase even further with continued R\&D.

We have also studied the natural recovery from radiation damage at room temperature, as well as the recovery in duced by thermal annealing and optical bleaching. Figur: 7 shows the absorbance spectrum measured during and immediately after irradiation with ${ }^{60} \mathrm{Co}$ gamma rays for a $1^{n}$ diameter $x 1^{n}$ long sample at room temperaturc. The data indicate no substantial difference in the shape of the absorbance curves measured during irradiation compared with those measured after, aithough we observe a slight recovery in the level of the absorbance on a time scale of a few minutes to several hours. This recovery can be greatly enhanced by either thermal annealing or optical bleaching. However, we found that thermal annealing to a temperature of $\sim 400^{\circ} \mathrm{C}$ for several hours in dry nitrogen produced physical changes in the crystal which reduced its transparency. On the other hand, optical bleaching proved to be much more effective and did not cause physical damage to the crystal. Figure 8 shows the recovery of an $18.6 \mathrm{~cm}$ block of $P b F_{2}$ as a function of exposure to light from a mercury lamp, passed through a $400 \mathrm{~nm}$ filter. The recovery of even such a large crystal is nearly complete after only 10 minutes of bleaching.

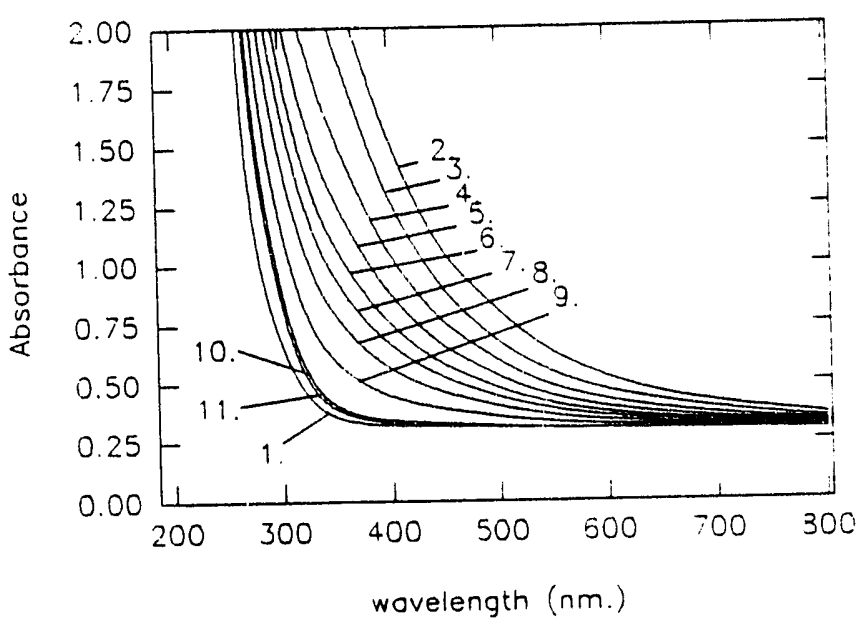

Fig. 8: Recovery in the transmittance of an 18.6 cm long $\mathrm{PbF}_{2}$ crystal with various exposures to light from a mercury lamp passing through a 400 nm filter. 1- initial, 2- immediately after 1 Mrad, 3- 20 days after 1 Mrad, 4- 15 sec. bleaching, 5- 30 sec., 6- 45 sec., 7- 60 sec., 8- 90 sec., 9- 150 sec., 10- 10 min., 11- $30 \mathrm{~min}$.

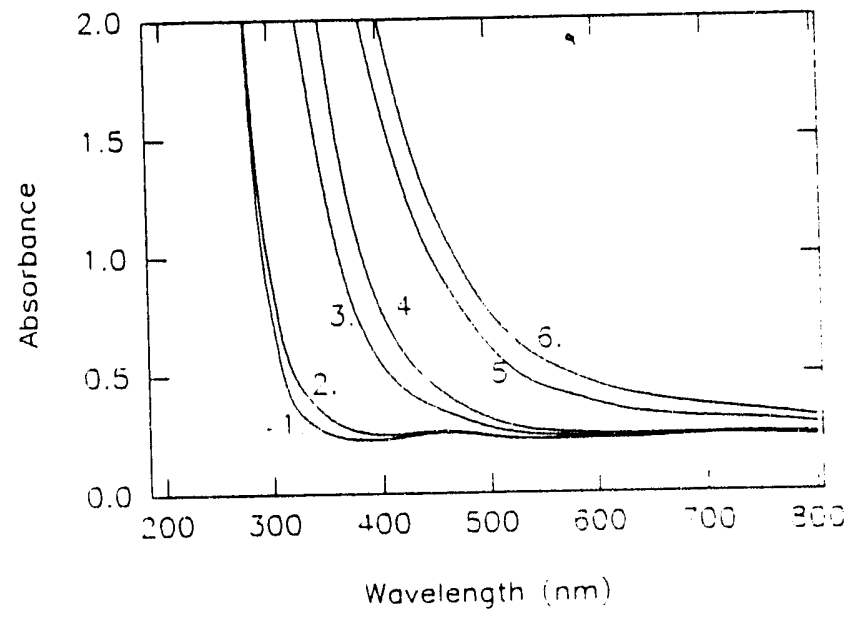

Fig. 8: Radiation damage in an $17.3 \mathrm{~cm}$ long $\mathrm{PbF}_{2}$ crystal caused by hadron irradiation at the AGS. Doses were measured in LiF dosimeter8 placed next to the crystal. 1- initial, 2- 0.8 Krad, 3- 13.5 Krad, 4- 42.3 Krad, 5- 390.2 Krad, 6- 654.2 Krad. The actual dose in in the $P b F_{2}$ may be 2-3 times higher (see text).

Figure 9 shows the damage produced in an $18.6 \mathrm{~cm}$ long $\mathrm{PbF}_{2}$ crystal due to an exposure to high energy hadrons. The sample was placed in one of the primary target caves at the Brookhaven AGS and exposed to a mixture of secondary particles produced by a $24 \mathrm{GeV}$ proton beam hitting a copper target. The dose given was measured in LiF dosimeters placed next to the sample during the irradiation, but the actual dose in the $P b F_{2}$ was 2-3 times greater due to the higher energy loss in 


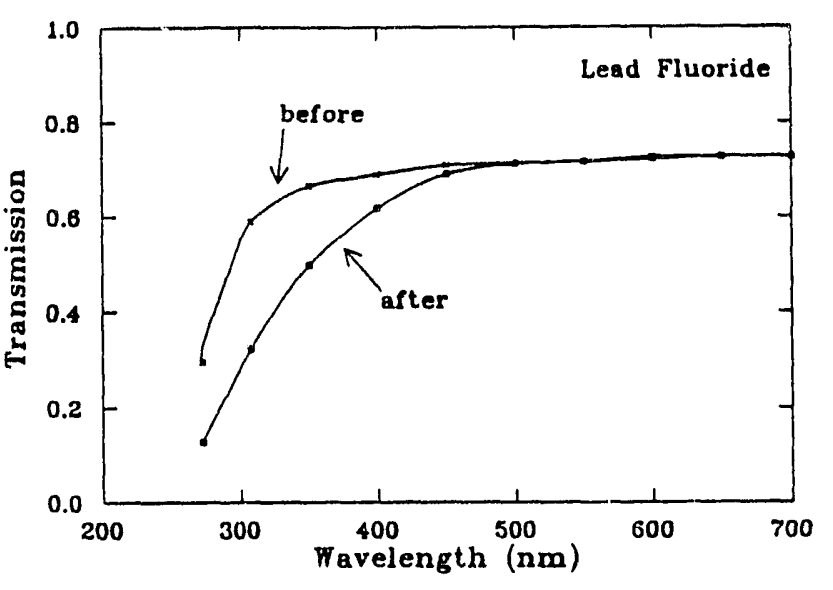

Fig. 10: Radiation damage in a 1 " diameter $x$ 1 " long $P b F_{2}$ sample after $125 \mathrm{Krad}$ of $300 \mathrm{MeV} / \mathrm{c}$ protons. Transmission measurements after irradiation were made several weeks after the exposure.

$\mathrm{PbF}_{2}$ at these energies. The absorbance measurements were typically made with 30 minutes after irradiation, although the exposure itself was not always continuous and some recovery could have taken place while the sample was being irradiated. The effects produced by hadron$s$ are similar to those observed with ${ }^{60} \mathrm{Co}$ irradiation and indicates only a modest amount of damage up to a level of a few tens of kilorads.

Figure 10 shows a $1^{n}$ dianieter $\times 1^{n}$ long sample irradiated to a dose of $125 \mathrm{Kred}$ with $300 \mathrm{MeV} / \mathrm{c}$ protons at TRIUMF [5]. In this test, the transmission was measured several weeks after irrsdiation, which could explain the lack of damage observed at longer wavelengths. However, in both the AGS and TRIUMF hadron irradiations, the damage observed is similar to that which has been observed with ${ }^{60} \mathrm{Co}$. It is therefore expected that the improved material now being grown will show better radiation resistance to hadrons as well as gamma rays.

Figure 11 shows the thermoluminescence spectrum observed in a small ( $5 \mathrm{mg}$ ) $P b F_{2}$ sample measured 15 minutes after irradiation to $1 \mathrm{Mrad}$ with ${ }^{60} \mathrm{Co}$ gamma rays. Two prominent glow peaks are seen at $\sim 100^{\circ} \mathrm{C}$ and $250^{\circ} \mathrm{C}$. However, the intensity of these peaks are extremely weak. They are approximately four orders or magnitude weaker than similar peaks which have been observed in, for example, barium fluoride. It is nevertheless likely that they are due to impurities or defects in the crystal and are related to those which give rise to radiation damage.

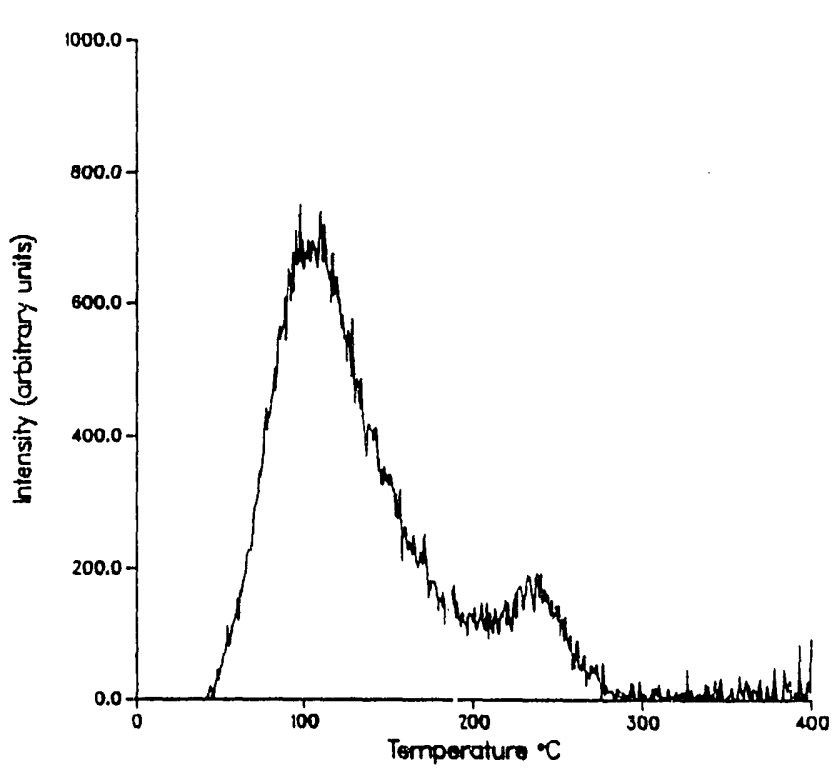

Fig. 11: Thermoluminescence spectrum measured in a small $P b F_{2}$ sample irradiated to $1 \mathrm{Mrad}$ with ${ }^{60} \mathrm{Co}$ gamma rays.

\section{CONCLUSIONS}

A prototype $\mathrm{PbF}_{2}$ calorimeter consisting of twenty five 20 radiation length crystals has been built and tested and shown to provide an energy resolution $\sim 5 \%$ at 1 $\mathrm{GeV}$. The number of photoelectrons per $\mathrm{GeV}$ has been measured to be $\sim 1000$ in good quality crystals, and could improve with better quality crystals now being grown. The Moliere radius has been found to be $\$ 2 \mathrm{~cm}$, and to give a position resolution on the order of a few millimeters. The $e / \pi$ ratio has been determined to be $\sim$ 1:100 at $2 \mathrm{GeV}$.

The effects of radiation damage have been measured and only modest effects are seen up to $\sim 30 \mathrm{Krad}$ in long crystals. The radiation damage can be thermally annealed or optically bleached, but thermal annealing can cause physical damage to the crystal. A weak thermoluminescence signal is observed after irradiation with gamma rays. Although the crystals tested still shows signs of damage from both gamma rays and hadrons, it is clear that the radiation hardness has been greatly improved over material which has been grown in the past.

In summary, $P b F_{2}$ has been shown to be an effective calorimeter material which can achieve an energy resolution which is comparable to or better than which can be obtained with lead glass. Large calorimeter sired crystals are now available and can provide a viable alternative to lead glass as a dense, highly efficient Cherenkov material. Moreover, the development of $P b F_{2}$ now appears to be at a stage where it can be considered for use in practical applications. 


\section{ACKNOWLEDGMENT}

This work was supported in part under Department of Energy Contract No. DE-AC02-CH7600016.

\section{REFERENCES}

1. D.F.Anderson et.al., Nucl. Inst. Meth. A290 (1990) 385.

2. E.B.Dally and R.Hofstadter, Rev. Sci. Inst. 39 (1968) 658;

E.B.Dally and R.Hofstadter, IEEE Trans. Nucl. Sci. NS-15 (1968) 76.

3. Optovac, Inc., North Brookfield, Ma., a division of Merck, Inc.

4. T.Awes et.al., Nucl. Inst. Meth. A311 (1992) 130.

5. Y.Kuno et.al., E787 Technical Note 222, Nov. 1991, TRIUMF, Vancouver, British Columbia, Canada.

\section{DISCLAIMER}

\footnotetext{
This report was prepared as an account of work sponsored by an agency of the United States Government. Neither the United States Government nor any agency thereof, nor any of their employees, makes any warranty, express or implied, or assumes any legal liability or responsibility for the accuracy, completeness, or usefulness of any information, apparatus, product, or process disclosed, or represents that its use would not infringe privately owned rights. Reference herein to any specific commercial product, process, or service by trade name, trademark, manufacturer, or otherwise does not necessarily constitute or imply its endorsement, recommendation, or favoring by the United States Government or any agency thereof. The views and opinions of authors expressed herein do not necessarily state or refiect those of the United States Government or any agency thereof.
} 

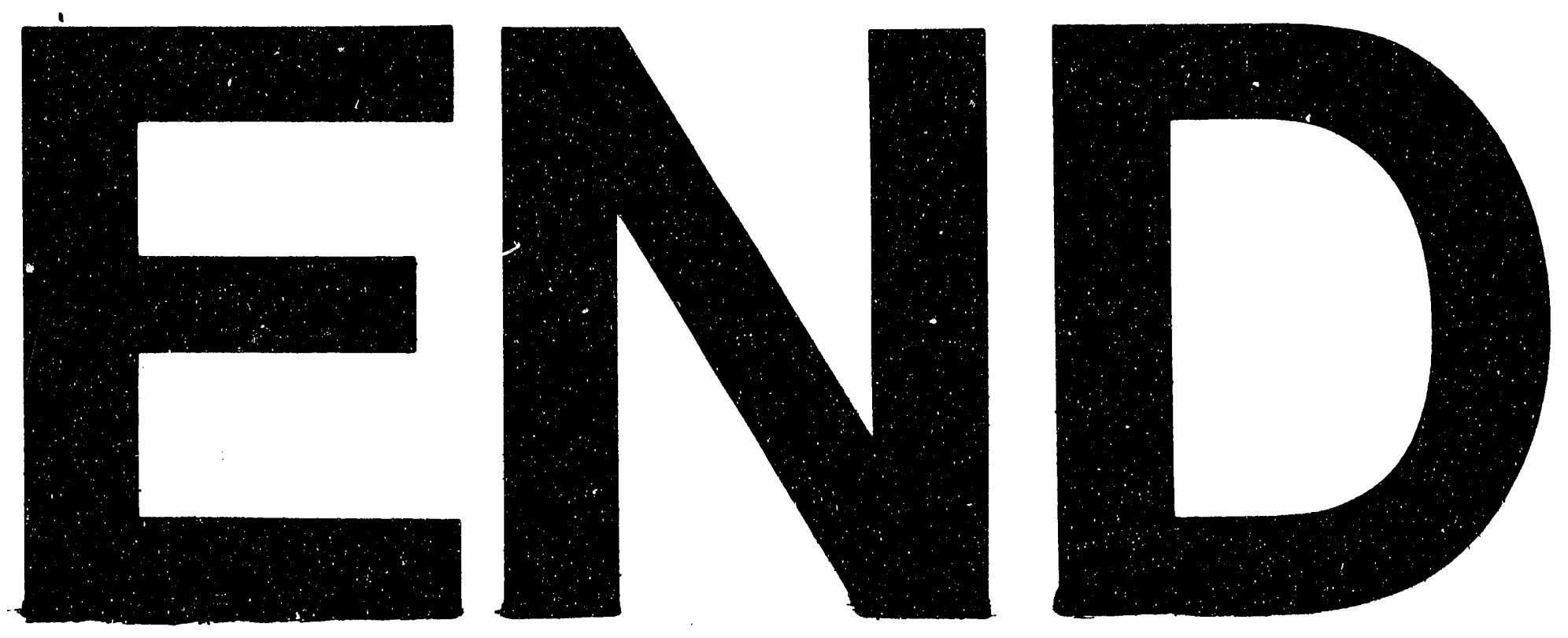

$x$
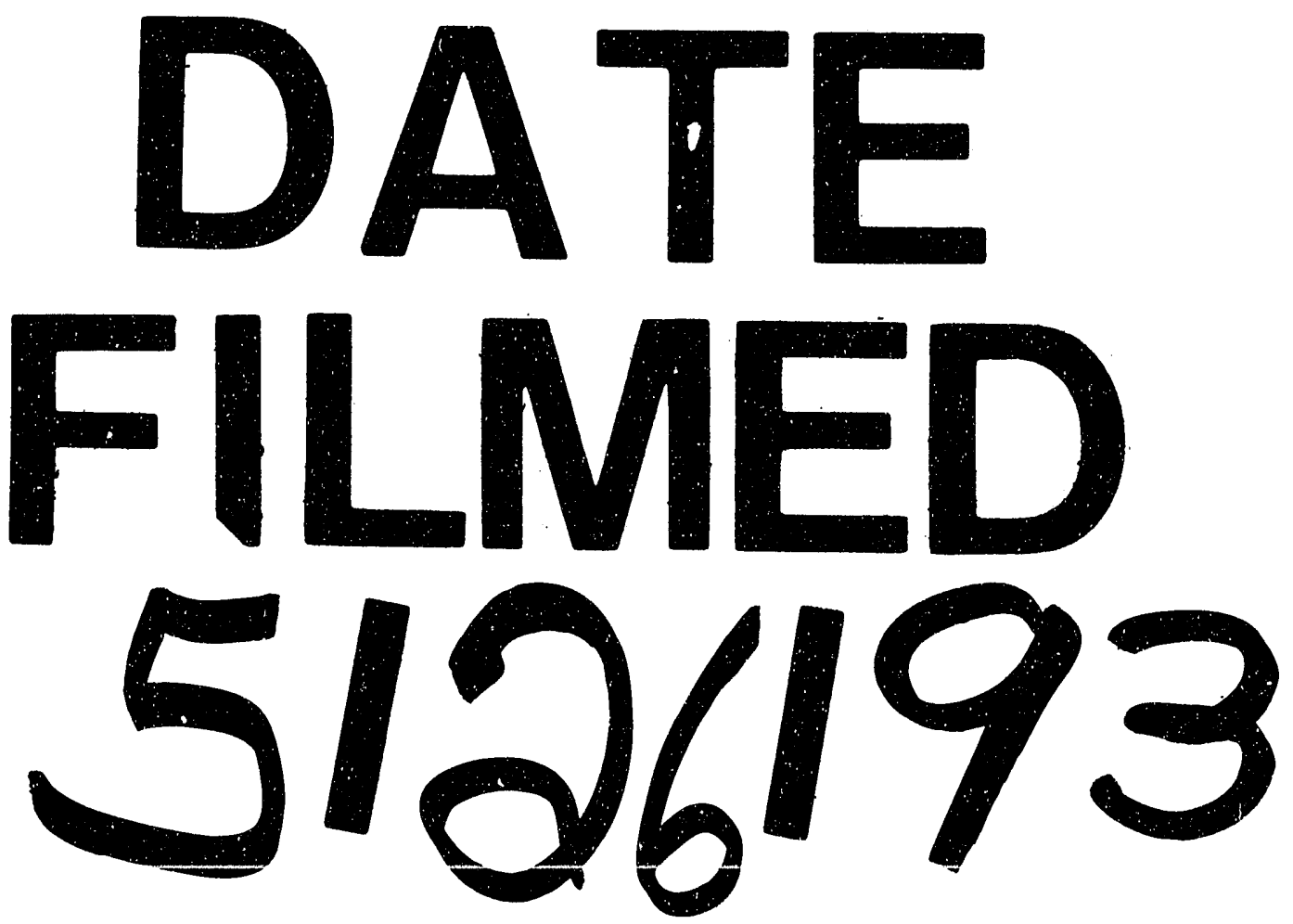


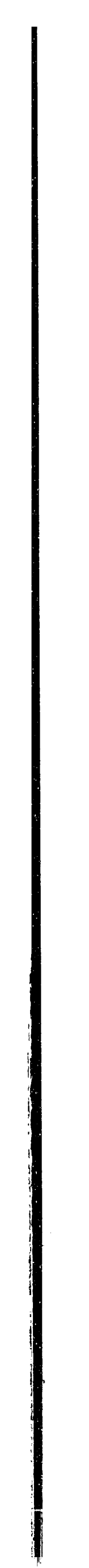

